\title{
« Ne pas leur faire confiance serait leur faire offense ». Antiterrorisme, solidarité démocratique et identité politique
}

\section{Emmanuel-Pierre Guittet}

\section{(2) OpenEdition Journals}

Édition électronique

URL : http://journals.openedition.org/conflits/2037

DOI : $10.4000 /$ conflits.2037

ISSN : 1777-5345

Éditeur :

CCLS - Centre d'études sur les conflits lilberté et sécurité, L'Harmattan

Édition imprimée

Date de publication : 1 mars 2006

Pagination : $51-76$

ISBN : 2-296-00622-1

ISSN : 1157-996X

Référence électronique

Emmanuel-Pierre Guittet, « « Ne pas leur faire confiance serait leur faire offense ». Antiterrorisme, solidarité démocratique et identité politique », Cultures \& Conflits [En ligne], 61 | printemps 2006, mis en ligne le 17 mai 2006, consulté le 30 mars 2021. URL : http://journals.openedition.org/conflits/2037 ; DOI : https://doi.org/10.4000/conflits.2037

Ce document a été généré automatiquement le 30 mars 2021

Creative Commons License 


\title{
« Ne pas leur faire confiance serait leur faire offense ». Antiterrorisme, solidarité démocratique et identité politique
}

\author{
Emmanuel-Pierre Guittet
}

1 Les attentats du 11 septembre 2001 sur le sol américain semblent avoir donné une mesure supplémentaire et une certaine urgence aux discours de la coopération internationale contre le terrorisme ${ }^{1}$. Suite à ces attentats, nombre d'hommes politiques, de praticiens et d'universitaires européens de tous horizons ont contribué à élever la lutte contre le terrorisme au niveau de la priorité des priorités en présentant le terrorisme comme la pire des menaces pour toute démocratie et en appuyant leurs prises de positions d'un élément commun: la nécessaire et naturelle solidarité démocratique contre le terrorisme. Ces attentats du 11 septembre 2001 semblent avoir ouvert un espace politique pour prétendre que toute politique de sécurité intérieure se doit d'être assurée au niveau mondial ou ne pas être assurée du tout. Cette solidarité naturelle des sociétés démocratiques européennes contre le terrorisme est un discours qui ne peine pas à s'imposer comme vrai, tant il s'énonce sur le mode de l'évidence. Evidence de la menace qui produit l'évidence de la réponse: une coopération antiterroriste de tous contre un ennemi communément admis.

2 Ce discours d'autorité à la coopération en matière de lutte contre le terrorisme s'énonce d'un ton grave et sentencieux à la mesure de l'imprécation à laquelle il renvoie. C'est parce que les Etats auraient collectivement découvert leur faiblesse face à la menace transnationale du terrorisme que tout un chacun s'est mû en acteur responsable d'une logique de tous contre une même menace débordant les frontières souveraines. C'est parce que le terrorisme serait une menace d'ordre vital contre toute démocratie ${ }^{2}$ que la coopération antiterroriste s'est faite vecteur d'action et témoignage spontané d'un intérêt commun à défendre un ensemble de valeurs collectives soumis à la pression d'une violence transnationale inacceptable. Véritable lieu commun 
rhétorique, ce discours d'autorité à la coopération internationale des démocraties européennes contre le terrorisme ne dit pourtant que peu de choses sur les relations précises qui existeraient entre une démocratie et la résistance à la terreur. Mais il engendre néanmoins une position normative forte: aucune démocratie digne de ce nom ne peut se refuser à la coopération sans prendre le risque de perdre derechef ses qualités démocratiques. En ce sens, la qualité de la coopération antiterroriste donnerait à voir les qualités d'une démocratie ${ }^{3}$.

3 C'est cette adéquation performative entre «démocratie» et «coopération» antiterroriste qui nous semble intéressante à analyser. L'idée d'un "terrorisme qui dépasse les frontières nationales et souveraines ", qui créerait le besoin d'une assistance mutuelle et d'une coopération sans faille, n'est pas une question qui peut être restreinte à la seule analyse de son efficacité fonctionnelle promise ou avérée. La force de ce discours d'autorité c'est que tout déchiffrement de la coopération antiterroriste européenne, à partir des formes dans lesquelles celle-ci s'est imposée, et donc à partir de sa nécessité pratique supposée, reconduit inévitablement au caractère indépassable de ses évidences. L'évidence empirique (l'ensemble de ces discours informant d'une solidarité née d'une heureuse et sincère concertation entre les Etats européens) et l'évidence fonctionnelle (l'ensemble des expériences politiques, des gains institutionnels et des réussites policières) invisibilisent très certainement la question de ce que l'antiterrorisme veut dire. La confusion entre les intentions exprimées, les systèmes de justification développés à la faveur de circonstances singulières (les attentats à New York le 11 septembre 2001, ceux de Madrid le 11 mars 2004, ou encore ceux de Londres le 7 juillet 2005) conduisent de nombreux analystes à ne pas se poser la question de savoir ce que le fonctionnement actuel de l'antiterrorisme européen doit à la manière dont celui-ci s'est constitué et imposé depuis plusieurs décennies comme lieu commun de la sécurité de tous, comme vecteur identitaire des démocraties européennes.

Comprendre l'antiterrorisme européen comme discours d'autorité à la coopération sans faille suppose, à notre sens, de se donner les moyens de retrouver les conditions de félicité de ce discours particulier et d'analyser les raisons de cette adéquation entre une formulation hyperbolique de la coopération antiterroriste (une règle du toujours plus et jamais assez) et la possibilité d'une représentation collective avantageuse de ce qu'être une démocratie européenne signifie. Au cœur de cet article, nous souhaitons démontrer comment la lutte antiterroriste en Europe a été initialement formée sur ce problème de la reconnaissance de chaque Etat membre comme membre inter pares de cette Europe des démocraties, et comment l'idée d'un déficit de sécurité engendré par le principe de la libre circulation dans la Communauté européenne a assuré la circulation de représentations permettant la conversion d'identités nationales en une identité collective forte : la démocratie pour tous les Etats membres.

5 Pour comprendre à la fois l'origine et l'efficacité de cette métamorphose identitaire à travers les enjeux de Sécurité, Liberté et Justice liés à la lutte contre le terrorisme, le cas espagnol nous paraît particulièrement approprié. Aucun autre Etat membre de l'Union européenne mieux que l'Espagne n'a su saisir l'occasion politique ouverte par les attentats du 11 septembre 2001 pour réaffirmer combien la lutte contre le terrorisme relevait de la priorité des priorités, en rappelant le conflit qui l'oppose à l'organisation clandestine basque ETA (Euskadi (e)Ta Askatasuna, "Pays basque et Liberté ») depuis des décennies. Il s'agit là précisément d'un élément fort du contexte 
normatif de la politique extérieure de l'Espagne qui, depuis la fin des années 1970, a toujours joint à sa demande d'intégration dans la Communauté européenne une réclamation de la nécessité d'une coopération mutuelle franche et entière en matière de lutte contre le terrorisme ${ }^{4}$. Après les attentats du 11 septembre 2001, les autorités espagnoles ont alors d'autant plus facilement accepté la rhétorique américaine de la "guerre contre le terrorisme » que celle-ci leur permettait à la fois de développer de nouvelles mesures antiterroristes, ou, tout du moins, de justifier leur dispositif antiterroriste des plus conséquents, en invoquant l'image d'une démocratie qui connaît à la fois la tragédie du terrorisme mais aussi l'importance de la solidarité internationale en la matière. De là, les autorités américaines ont trouvé un allié européen précieux dans les autorités espagnoles tandis que l'Espagne a trouvé un discours international qui lui a permis de légitimer son problème interne du Pays basque. Par ailleurs, si l'on prend acte des inscriptions d'individus sur la liste européenne des terroristes ${ }^{5}$, on constatera aisément l'implication espagnole en la matière. Sur une liste de quarantecinq individus, dix-neuf membres de l'organisation clandestine basque ETA ont été inscrits par les autorités espagnoles. De plus, il convient de considérer combien la décision du Conseil de l'Union européenne relative à la mise en place, le 13 juin 2002, d'un Mandat d'arrêt européen (MAE) visant à remplacer les anciennes mesures d'extradition est une réponse à une revendication espagnole déjà ancienne ${ }^{6}$.

6 En prenant le cas espagnol à travers une relecture de l'histoire de l'organisation clandestine antiterroriste espagnole des GAL (Groupes antiterroristes de libération), des conditions socio-politiques de la signature du Protocole sur le Droit d'asile des ressortissants des Etats membres de l'Union européenne (dit «Protocole Aznar ») et de la mise en place du MAE, nous pouvons retrouver à la fois les conditions sociohistoriques de la genèse et de l'impact de ce discours d'autorité européen à la coopération comme impératif de la sécurité de tous, comme nous pouvons aussi comprendre, dans le même mouvement, comment celui-ci s'est forgé sur une représentation collective avantageuse de ce qu'être une démocratie européenne veut dire. Autrement dit, nous prétendons joindre dans cet article deux éléments toujours tenus à bonne distance l'un de l'autre: la sécurité et l'identité collective. La problématique de la sécurité et la problématique de l'identité sont les deux faces d'un même processus, celui de la construction européenne. Au fur et à mesure que le discours de la coopération naturelle fait de plus en plus sens pour les acteurs de la sécurité européenne, celui-ci se renforce et soutient le discours de la démocratie comme première et unique victime du terrorisme. C'est donc l'histoire d'un processus social et historique complexe d'identification que nous souhaitons mettre en perspective : comment la définition commune d'une situation (le terrorisme comme menace) se construit sur un référent commun, à savoir le déficit de sécurité, s'imposant à travers la problématique de la libre circulation, et ouvre de la sorte à un espace commun de significations politiques propres à dire l'identité de l'ensemble et de chacune des parties de cet ensemble. Pour ce faire, il faut contourner la coopération comme discours, comme programme de vérité pour l'interroger dans sa constitution historique.

7 Il y a un discours politique comme il existe un discours technique sur les moyens à employer en termes de coopération antiterroriste. Ce dernier dit très clairement les «difficultés» de la coopération, et le premier les « raisons » de cette même coopération. Nombreuses sont les publications sur le sujet. Mais, malheureusement, la plupart de ces ouvrages, qui tentent d'analyser les pratiques policières, la coopération juridique ou 
encore la place et les enjeux des services de renseignement en la matière sont bien souvent construits de la même manière et respectent la doxa, cette mise en mots de l'histoire officielle de la coopération antiterroriste européenne qui commence par la fin des années 1970, où la nécessité de la recherche d'un « remède » collectif à la criminalité internationale serait devenu un incontournable des discussions policières et politiques. Un point de départ à la coopération de tous qui répondrait à une gravité d'autant plus grande que l'Union européenne, comme espace de liberté de circulation pour les marchandises, les capitaux et les personnes, offrirait un environnement des plus propices à la "propagation du crime ». L'achèvement du Marché unique se serait donc tout naturellement accompagné d'une mobilisation sans précédent de la part des Etats membres pour lutter de concert contre la criminalité transfrontalière et, parmi celle-ci, le terrorisme. Ce souci de sécurité, présenté comme mesure corollaire à l'instauration du Marché unique, tiendrait de l'évidence, tant il est vrai qu'il y avait urgence à organiser une réponse collective à une menace collective. La plus grande partie de ces publications part de ce même point et suit ce discours politique sur les « raisons » de la coopération : ce seraient les exactions terroristes des années 1970 et 1980 qui auraient poussé les Etats à prendre conscience que l'identité des valeurs fondamentales permettait une confiance réciproque. En ce sens, ces publications participent ou soutiennent (pour des raisons idéologiques ou par ignorance) les justifications de ce discours politique à la coopération ${ }^{7}$. Le problème principal de ces publications est qu'elles ne perçoivent pas les dynamiques structurelles (nationales ou européennes), les processus et les stratégies (conscientes ou inconscientes) qui sont profondément ancrés dans des environnements politiques et historiques nationaux spécifiques. Ces mêmes publications tendent aussi à ignorer ou minorent l'importance de ce discours d'autorité à la coopération comme construction même d'un lieu commun pour dire la sécurité de tous, alors que nous sommes devant un superbe phénomène de causalité circulaire où les besoins de la coopération (réelle ou imaginée) détermineraient les nécessités en la matière, celles-ci déterminant en retour les besoins de la coopération. En d'autres termes, l'augmentation de la coopération exige toujours une nouvelle étape dans la coopération, suivant un mouvement perpétuel du toujours plus, jamais assez.

8 La coopération n'est pas une formule de solidarité pure sans contrepartie. La règle de la coopération est fondée sur l'échange. Cet échange permet l'entretien de la cohésion sociale, précisément en transformant les vieilles rivalités et autres malentendus en objectifs communs. Mais il est également un échange relatif à l'identité qui permet la résolution des contradictions les plus importantes de notre époque actuelle. C'est-àdire la contradiction entre une nouvelle formation sociale et économique sans frontières (la globalité, au sens d'Ulrich Beck) et encore plus de formations politiques spécifiques, où les frontières sont à la fois une configuration nécessaire et une modalité d'affirmation. La coopération pourrait être perçue comme paradoxe. Elle est autant une formule de salut pour les Etats, qu'une possibilité d'une raison d'Etat plurielle pour chacun où la question des pratiques violentes serait consciencieusement laissée de côté au nom du principe de la reconnaissance mutuelle de la démocratie entre Etats de l'Union européenne ${ }^{8}$. La violence d'Etat ne peut pas exister au sein de l'Union européenne. L'identité européenne comme identité démocratique permet de recouvrir l'illégalité suivant une politique de l'amnésie qui convertit le plomb de la coopération policière en or de la qualité démocratique de l'Etat de droit. L'histoire et l'oubli des GAL comme organisation antiterroriste clandestine parrainée par l'Etat espagnol dans les années 1980 pour lutter contre l'ETA sur un territoire voisin, la France, est un épisode 
important pour comprendre à la fois la genèse de la lutte contre le terrorisme en Europe, produire une vue différente sur la légitimité, la sécurité, la démocratie et l'Europe, et rendre compréhensible cette jonction entre "démocratie " et "lutte antiterroriste ".

Que savons-nous des GAL? Tout d'abord que les GAL sont une création de plusieurs hauts responsables politiques espagnols, parmi lesquels Ricardo Garcia Damborenea, Secrétaire général du PSOE en Euskadi ${ }^{9}$ au moment des faits, qui reconnaît, en 1995, lors de sa mise en accusation, être à l'initiative de l'organisation, et Julian Sancristobal Iguaran, gouverneur civil de la province de Viscaye, qui reconnaît être l'auteur du sigle. Nous sommes ici face à des responsables politiques sous l'influence des analyses des services secrets espagnols du CESID, avec qui ils étaient en liaison, plus ou moins directement et plus ou moins officiellement. Le recrutement de la cinquantaine de mercenaires, reconnus ou suspectés dans l'affaire, est à imputer aux services de la policía et de la Guardia civil espagnoles, en place à Bilbao ${ }^{10}$ et à San-Sebastian ${ }^{11}$. Quant au financement, il émane du ministère de l'Intérieur, puisant sur les fonds secrets mis à sa disposition et à sa discrétion. Enfin, rappelons que les trente-cinq opérations connues des GAL, ont toutes eu lieu sur le sol français, entre Bayonne, Hendaye et Saint-Martin d'Arrossa, hormis le double assassinat de Lasa et Zabala (le 16 octobre 1983 à SanSebastian), l'assassinat de Santi Brouard (le 20 novembre 1984 à Bilbao) et la séquestration de Segundo Marey (le 14 décembre 1983 à Bilbao) ${ }^{12}$.

Pourquoi s'intéresser aux GAL, à ces Groupes Antiterroristes de Libération qui, sous couvert d'un financement sur les fonds secrets du gouvernement socialiste espagnol et avec l'appui des institutions de sécurité ibériques, employèrent des mercenaires pour assassiner plus d'une vingtaine de membres de l'ETA dans le sud de la France de 1983 à 1987 ? Voilà une organisation clandestine patronnée par un Etat qui prétend à la qualification d'antiterrorisme et qui, in fine, est condamnée au terme de longues procédures par les tribunaux espagnols et français comme «terroriste». Durant la campagne d'attentats et d'assassinats des GAL de 1983 à 1987, ce ne sont pas moins d'une trentaine de mercenaires qui sont arrêtés par la police française pour " appartenance à groupe armé " et jugés comme tels. Pas moins de quinze hauts fonctionnaires d'Etat espagnols, dont un ministre de l'Intérieur, José Barrionuevo, sont condamnés par la justice espagnole pour la constitution et le financement des GAL.

11 Les GAL sont une organisation clandestine dont les membres sont condamnés au même titre que les membres d'une autre organisation clandestine, l'ETA. La question de savoir si l'antiterrorisme et le terrorisme se ressemblent n'est pas dénuée de fondement dans cette configuration violente particulière. Ce qui nous paraît important pour l'étude des GAL, comme point de départ de notre travail empirique à la compréhension de la force de ce discours de la coopération antiterroriste, c'est qu'au moment même où les GAL agissent, il existe aussi par ailleurs un antiterrorisme officiel. Or, on ne peut passer sur l'inventaire de ce que ces deux logiques d'antiterrorisme peuvent avoir en commun et de différent. L'antiterrorisme clandestin fonctionne à l'intérieur de l'antiterrorisme officiel comme il s'y oppose. Il fonctionne aussi en parallèle et de manière convergente pour une raison d'ordre généalogique: ces deux logiques, ces deux formes d'antiterrorisme se nourrissent des mêmes éléments, du même contexte sociohistorique, des mêmes acteurs et des mêmes visées. Pour en finir avec l'ETA et forcer les autorités françaises à modifier leur point de vue sur la situation de leurs réfugiés basques, la règle est celle de "la fin justifie les moyens", y compris le retour aux 
pratiques parapolicières employées sous la dictature franquiste: attentats contre attentats, assassinats contre assassinats. Ce que l'on constate, du moins avec le cas des GAL, c'est qu'il existe de vraies formes d'intimités entre les deux logiques d'antiterrorisme. Que ce soit la lutte contre l'ETA, l'enjeu de la coopération avec les autorités françaises et l'entrée de l'Espagne dans l'Europe, l'antiterrorisme clandestin comme l'antiterrorisme officiel agissent pour et en fonction de ces problématiques. Les griefs de l'Espagne sont nombreux à l'égard du voisin du nord, la France. Le reproche de coupable indulgence à l'égard du "terrorisme " basque est très certainement le premier. C'est donc en cela que, lorsque les GAL commettent des actions hors du territoire espagnol, ils se tournent certes vers les membres de l'ETA réfugiés au-delà de la frontière des Pyrénées, mais aussi contre les autorités françaises dans une forme de défi, de pied de nez à la situation : la frontière franco-espagnole est à la fois poreuse pour les commandos de l'ETA comme pour ceux des GAL.

Convoqué par l'Audiencia Nacional à Madrid, pour apporter son témoignage dans le procès, au titre d'ancien directeur de la Guardia civil de 1983 à 1986, sur la manière dont les fonds secrets gouvernementaux ont été utilisés dans la lutte antiterroriste des années 1980 en Espagne, le Général José Antonio Sáenz de Santamaría n'a pas d'hésitation à déclarer que :

« la lutte antiterroriste ne peut pas être poursuivie dans le cadre de la loi. A cet égard, nous sommes plus souvent au bord de la loi, en dehors de la loi et de temps à autre dans le cadre de la loi... mais il n'y a aucune autre manière que d'utiliser la guerre irrégulière contre ces types qui vous tirent dans le dos. La règle de la loi est une bonne chose mais nous ne pouvons pas définitivement la respecter parce qu'elle fait le jeu des terroristes ${ }^{13}$ ".

Les GAL mettent en perspective cette idée selon laquelle l'antiterrorisme peut passer outre les règles communes de droit et imiter l'ennemi dans la lutte contre celui-ci. Ce désir interdit, la constitution d'un double à l'organisation clandestine de l'ETA, prend forme dans la relation conflictuelle, structurée par les jeux de rivalité et de théâtralisation, où la diabolisation de l'adversaire, l'ETA, vient en quelque sorte légitimer l'usage des mêmes armes que la contre subversion lui attribue, mais au nom cette fois de la nécessité supérieure - Raison, nécessité et Sécurité d'Etat - de mettre en échec les plans de la subversion. Il y a quelque chose de l'ordre de la symbiose entre la contre subversion et son ennemi désigné. Et les GAL ne sont que la formalisation extrême de cette rivalité mimétique entre l'ETA et les pouvoirs publics espagnols, où la nécessité d'agir par tous les moyens contre l'organisation clandestine rend la formation des GAL possible en même temps que souhaitable. Une rivalité mimétique jusque dans le détournement du slogan guerrier et de la signature de l'ETA. «Bietan Jarrai ». Cela signifie «les deux voies" dans la langue basque. Cette formule se révèle dans la signature de l'ETA : le serpent lové (la ruse) autour d'une hache (la force). Les GAL ont détourné ce symbole pour signer et signifier leurs actions: la tête du serpent est coupée par la hache. Les GAL ont choisi la force sur la ruse pour en finir avec l'ETA ou, tout du moins, pour pousser les autorités françaises à reconsidérer leur politique en matière d'extradition en agissant directement en France, dans le «sanctuaire » (la tête) de l'ETA.

14 L'attitude bienveillante des autorités françaises vis-à-vis des réfugiés basques, une certaine méfiance à l'égard de la politique espagnole, voire une certaine forme de condescendance de la part des autorités politiques françaises face à la «jeune démocratie » ibérique, tout comme le discours français du respect de l'asile politique 
participent très certainement à l'obstacle pour les autorités espagnoles en quête de partenaires et partisans de l'abolition du «sanctuaire " français en particulier et de l'asile politique en Europe en général. Or, au moment où l'Espagne, après la mort de Franco, s'efforce de rejoindre la Communauté européenne, pour les autorités françaises le droit d'asile et l'extradition de citoyens espagnols d'origine basque sont antinomiques. Ceux-ci ne souffrent aucune discussion et, quand bien même l'ETA est considérée, depuis 1972, comme une "association étrangère illégale » en France ${ }^{14}$ : on n'extrade pas les réfugiés basques. L'extradition est fondamentalement une question politique qui engage deux gouvernements. En la matière, à cette époque, la position française a été largement conditionnée par la "principale préoccupation» d'éviter tout attentat sur son sol $^{15}$. Au grand dam des autorités espagnoles, la question d'une «nécessaire » solidarité politique envers la démocratie espagnole était alors beaucoup moins importante que l'adoption d'une position neutre: le problème basque reste un problème espagnol. A ce discours pragmatique se joint aussi une haute considération pour ce que certains appellent la tradition française d'asile politique. Or, pour ces mêmes acteurs, l'Espagne était loin de posséder les qualités démocratiques nécessaires à une politique d'extradition ${ }^{16}$. C'est dans ce contexte politico-diplomatique que les GAL agissent, en même temps que les autorités espagnoles n'ont de cesse de réitérer leurs demandes officielles d'extradition des réfugiés basques.

Dès lors, qui de l'antiterrorisme clandestin et de l'antiterrorisme officiel est sorti vainqueur? Les GAL avaient promis de rendre coup pour coup à l'ETA et prétendaient en finir non seulement avec l'organisation clandestine basque, mais aussi avec la politique française d'accueil des réfugiés ${ }^{17}$. L'argument décisif en faveur de l'asile politique, tel qu'il est développé en France, est que l'acceptation de ce principe engageant à un refus d'extrader ne doit pas être vu comme une forme de sympathie envers les causes qualifiées de "politiques" pour la personne demandée, mais bien plutôt comme un acte de neutralité et de non-ingérence dans les affaires intérieures d'un autre Etat. En 1986, en pleine actualité des GAL, les autorités françaises procèdent aux premières extraditions vers l'Espagne de membres de l'ETA et depuis, les extraditions se sont poursuivies. Les autorités françaises extradent, mais l'ETA n'a pas pour autant disparue, voire s'est trouvée renforcée par les agissements des GAL ${ }^{18}$. S'agit-il d'une victoire en demi-teinte des GAL ? Les GAL n'en ont certes pas terminé avec l'ETA, mais les agissements de cette organisation antiterroriste clandestine cessent lorsque la France change de politique en matière d'extradition. Dans son ouvrage sur ses années auprès de la Présidence de la République française, Gilles Ménage s'insurge contre cette mise en perspective et estime que ce n'est, ni plus ni moins, qu'une ineptie ${ }^{19}$. Pourtant, il s'agit de la ligne de défense des acteurs politiques et des hauts responsables des institutions de sécurité espagnole lorsqu'ils font recours de leurs condamnations devant le Tribunal constitutionnel espagnol. Les GAL ont été créés pour "donner une leçon aux Gaulois $^{20}$ " et les obliger à coopérer en renvoyant vers l'Espagne les membres de l'ETA réfugiés sur leur territoire à un moment où la France s'y refusait ${ }^{21}$.

16 L'organisation clandestine écran des GAL a disparu parce qu'elle n'avait plus de raison d'être : la France extrade les réfugiés basques. Et quand le judiciaire s'en mêle, les GAL révèlent après plusieurs années de procédure leurs commanditaires inavouables et deviennent l'objet d'un scandale sans précédent en Espagne. Personne, et surtout pas les autorités françaises, ne souhaiteraient rouvrir la boîte de Pandore de ces escadrons de la mort financés et orchestrés par une démocratie voisine sans être contraints alors 
d'examiner les éventuelles implications de policiers français dans les $\mathrm{GAL}^{22}$ et de reconsidérer la question de la genèse de l'antiterrorisme en Europe sous cet angle peu soucieux des règles de l'Etat de droit. Les GAL doivent rester dans le placard de la coopération européenne parce qu'ils soulignent parfaitement combien la lutte contre le terrorisme constitue cette frontière où l'Etat de droit hésite perpétuellement entre luimême et son autre : l'Etat de police. Les GAL sont-ils autre chose que la réaffirmation d'un droit de soustraction politique à la loi en matière de lutte antiterroriste répondant à l'invocation de la nécessité de défendre, coûte que coûte et par tous les moyens y compris illégaux, la "démocratie en danger "? Confronté à la violence radicale, le champ d'action de l'Etat n'est plus soumis à aucune règle sinon à celle, "hors du droit ", que lui dictent les objectifs immédiats d'une politique qui se doit d'être celle de l'action et non de l'inaction, et qui se pense suivant un ensemble de croyances que les hommes politiques prêtent aux citoyens apeurés et terrifiés ${ }^{23}$.

De ce point de vue, c'est moins le manque ponctuel et structurel de coopération qu'il est important d'analyser que les discours qui stigmatisent le manque de coopération ou, à l'opposé, acclament les « succès » attribués à l'efficacité de la coopération où tout le monde est invité à réaliser combien cette mise à l'index du «terrorisme » est désormais l'affaire exclusive des démocraties. La coopération confère à l'Europe communautaire - au sens anthropologique - sa valeur politique. C'est vers elle qu'il faut se pencher pour atteindre le fond du problème de l'identité de l'Europe des Etats membres. La coopération permet un nivellement et une homogénéisation au moins dans les justifications, garantissant l'amnésie des pratiques déloyales et illégales de chacun.

Dans leur bataille contre les formes violentes et radicales du nationalisme basque, les autorités espagnoles, de part et d'autre de l'échiquier politique, n'ont eu de cesse, d'une part, de militer pour une application extensive de leur politique répressive de punition (à l'instar de l'épisode des GAL) et de leur politique d'extradition à l'égard des membres de l'ETA auprès des autorités françaises, et, d'autre part, d'entreprendre au niveau des institutions européennes des démarches permettant l'adoption "urgente et nécessaire " d'une position cohérente et commune dans l'application du droit d'extradition et du droit d'asile. La tentation espagnole d'en finir avec le concept d'infraction politique en matière extraditionnelle est une politique tous azimuts mais relativement constante dans ses fins. La succession des rencontres politiques entre la France et l'Espagne depuis l'orée des années 1980 est explicite de ce mouvement en faveur de la dépolitisation des actes et du renforcement de la coopération judiciaire comme exercice et marque de confiance réciproque. Or, pour les autorités espagnoles, cette position cohérente et commune passe par le choix à faire entre la tentative d'une définition plus précise de l'infraction politique ou par la disparition purement et simplement de ce concept.

19 C'est à proprement parler cette configuration politico-diplomatique particulière qui retiendra ici notre attention. Comment les autorités espagnoles, et cela depuis la fin des années 1970, ont-elles réussi à imposer la problématique de l'extradition comme point central dans la lutte antiterroriste et ont, ainsi, fortement contribué à métamorphoser les enjeux, les pratiques et les discours de la coopération en matière de lutte contre le terrorisme au point où l'organisation clandestine basque ETA est désormais inscrite au frontispice des listes terroristes européennes comme problème de l'Union européenne, comme ennemi commun? Dans l'axiome du discours de la menace qui veut que la 
criminalité internationale soit désormais le problème de tous, ne peut-on pas lire en filigrane combien l'identité démocratique de chacun dépend de la reconnaissance de l'autre comme démocratie? "L'asile ne vaut que pour les citoyens de pays tiers qui n'ont pas d'autre choix que de combattre une dictature par des moyens terroristes, et en aucun cas pour ceux qui combattent de la sorte un régime démocratique ${ }^{24} »$. La mise à nu de l'asile politique dans l'Union européenne pourrait être alors lue comme l'aboutissement logique de cette confusion générique dans les discours politiques européens de la démocratie, de l'Etat de droit et de la Raison d'Etat, sous prétexte que l'appartenance à l'Union supposerait, par principe, l'adoption exigeante et inconditionnelle d'une solidarité sans faille: «ne pas faire confiance, c'est faire offense ».

Conjointement aux Accords de Schengen lors de la Conférence Intergouvernementale de $1996^{25}$, la proposition de la part de l'Espagne d'une clause interdisant l'asile politique à un citoyen de tout autre Etat membre de l'Union européenne était directement liée à un litige diplomatique en cours avec la Belgique, à propos de l'extradition de deux citoyens espagnols, à savoir Luis Moreno Ramajo et sa femme Raquel García Arranz, tous les deux soupçonnés de complicité avec l'organisation clandestine basque ETA. "Personne ne peut permettre ou tolérer que le statut de réfugié politique soit appliqué à deux terroristes ${ }^{26} »$. Au moment où les GAL sont devenus un problème judiciaire et un scandale politique sans précédent en Espagne, le refus belge d'extrader deux membres présumés de l'ETA, fondé sur des doutes quant à la demande des autorités espagnoles, n'est plus acceptable pour le nouveau gouvernement de droite dirigé par José Maria Aznar. Dans ses mémoires d'ex-ministre socialiste de l'Intérieur, José Barrionuevo met l'accent sur cette histoire: "la bataille des extraditions, très dure et très difficile par moments, a été gagnée. Pour cette raison, cela a produit de l'étonnement quand au début de 1996 les autorités belges rejetèrent la demande espagnole d'extradition de deux etarras. Quelque chose devait aller de travers parce que, comme nous l'avons vu, les premières extraditions avaient été concédées précisément par la Belgique douze ans auparavant ${ }^{27}$ ». Pour les autorités espagnoles, il est injuste d'être isolé de la partie à cause de doutes sur leur attitude politique envers le problème basque. Il est aussi surtout essentiel pour les autorités espagnoles de gauche comme de droite, au pouvoir ou dans l'opposition, de mettre à l'ordre du jour national et européen non seulement l'extradition de ces deux membres présumés de l'ETA, mais aussi le fait de se donner les moyens pour que cet épisode ne puisse se renouveler ${ }^{28}$.

21 La question d'accepter ou non l'extradition d'un basque, réfugié pour les uns et terroriste pour les autres, constitue un problème politique et diplomatique d'envergure. En effet, reconnaître la qualité de prisonnier politique à une personne considérée comme terroriste en Espagne, cela revient à reconnaître implicitement à cet individu la qualité qu'il revendique et que l'Etat demandeur lui dénie. Pire, par effet d'extension, le refus d'une extradition est aussi relégation de la légitimité de la demande et, par conséquent, dénégation de la qualité démocratique de l'Espagne, à un moment où la transition politique réussie est le maître mot de l'identité de la politique étrangère espagnole, comme passeport pour l'entrée dans l'Europe communautaire ${ }^{29}$. Lorsque le gouvernement espagnol présente la menace de l'ETA comme faisant partie intégrante $\mathrm{du}$ problème de terrorisme transfrontalier, d'autres gouvernements européens, comme le gouvernement britannique ou le gouvernement allemand, écoutent de manière attentive ce discours et, concernant l'affaire Moreno-Garcia, c'est le Gouvernement belge qui se trouve ainsi ciblé. La rencontre entre José Maria Aznar et le Président français Jacques Chirac, à Paris en juin 1996, fut l'occasion pour le chef du 
gouvernement espagnol de souligner quelles sont les qualités essentielles d'une procédure d'extradition en rappelant combien la coopération franco-espagnole s'appuie sur cette logique de confiance mutuelle qui fait défaut par ailleurs : «Naturellement je dois dire que la coopération franco-espagnole fonctionne; elle fonctionne dans ce domaine, et va continuer à être renforcée. [...] Personnellement, comme vous le savez déjà, il me paraît inacceptable que des demandes d'asile de personnes ou d'autres difficultés en matière d'extradition puissent être problématiques parmi les membres associés qui forment l'Union européenne ${ }^{30} »$.

Au-delà de cette réaffirmation symbolique de la «nature " démocratique des Etats de l'Union européenne, obligés de rester aveugles faces aux pratiques de violence légitimées par l'Etat espagnol, ce propos structure un discours de coopération policière et judiciaire comme étant un objectif légitime.

"Le terrorisme est une menace pour tous, sans distinction de lieu, comme le montrent les attentats d'Oklahoma City et du World Trade Center à New York, les attentats à la bombe dans le métro de Paris et les dernières actions de l'IRA à Londres ainsi que de l'organisation terroriste ETA en Espagne. [...] Il faut, par conséquent, une impulsion au plus haut niveau politique. Au sein de l'Union européenne, nous tentons de développer une coopération extensive dans ce domaine. Malheureusement, le processus n'a été que très lent, même après que le traité de Maastricht ait identifié les domaines de coopération et établi de nouveaux mécanismes d'action. Entre-temps, l'activité terroriste et criminelle s'étend à vive allure. [...] Au-delà de l'échange des informations et des initiatives conjointes de formation, la coopération est essentielle au sein des autorités judiciaires. [...] Il faudrait aussi inclure le renforcement ou la simplification des mécanismes internationaux d'extradition et de transfert, afin de s'assurer que les criminels et les terroristes ne puissent trouver d'endroit où se cacher ${ }^{31}$ ".

Le fait que l'efficacité de la lutte contre le terrorisme doive passer par l'adoption de mesures permettant la mise en place d'un espace judiciaire européen fut rappelé sans cesse par les autorités espagnoles au fur et à mesure que le différend avec les autorités belges prenait de l'ampleur. Toujours est-il que ce sérieux différend hispano-belge réactive les travaux sur l'extradition au sein de l'Union et débouche sur l'élaboration d'une Convention relative à l'extradition entre les Etats membres ${ }^{32}$. Présentée comme le fruit d'une nécessité d'améliorer la coopération judiciaire entre les Etats membres de l'Union européenne pour les affaires criminelles, cette Convention, qui reposait sur l'article K.3 du traité de l'Union européenne et abordait l'extradition entre les Etats membres, fut ouverte à signature le 27 septembre $1996^{33}$. Cette Convention institue une modification fondamentale par rapport à la précédente Convention européenne d'extradition de 1957. Alors que celle-ci stipulait l'exclusion du champ d'application de l'extradition de toute infraction considérée par l'Etat requis comme relevant d'une infraction politique, la Convention de 1996 consacre le principe inverse : désormais, aucune infraction ne peut être considérée par l'Etat requis comme relevant d'une infraction politique. La Convention du 27 septembre 1996 modifie un peu plus le droit de l'extradition entre les Etats membres de l'Union en « détricotant » encore davantage l'exception traditionnelle à l'extradition pour infraction politique ${ }^{34}$. Résultat d'un compromis, obtenu après de longues et difficiles négociations afin de mettre un terme à une impasse politique entre deux Etats membres, cette Convention d'extradition de 1996 - qui ne rénove pas pour autant la procédure d'extradition, dans la mesure où elle ne remplace pas les instruments existants mais les complète ${ }^{35}$ - est la première 
concrétisation des revendications espagnoles présentées à la suite de l'affaire GarciaMoreno $^{36}$. Reste la question de la reconnaissance démocratique.

Le discours de la députée espagnole Ana Palacio Vallelersundi et les débats de la session du 18 septembre 1997 qui s'ensuivirent au Parlement européen sont d'un vif intérêt pour la compréhension de cette bataille diplomatique entre l'Espagne et la Belgique, perçue comme un problème commun à tous les Etats membres de l'Union européenne. Ana Palacio était à cette époque rapporteur pour le Comité des Libertés civiques et Affaires internes et devait présenter un rapport face à la proposition du Conseil pour une résolution de la Convention européenne d'extradition. Elle débute son discours en soulignant le manque de "vraie coopération» entre les Etats membres de l'Union européenne. Cette lacune serait une "vraie frustration" car, "ce que les Européens demandent c'est de simplifier l'extradition». Et, dans un Marché commun sans frontières, améliorer la coopération légale du droit pénal est essentielle pour accélérer le combat contre le terrorisme et le crime organisé. La simplification des conditions d'extradition fut avérée "grâce à l'étendue de l'intégration politique obtenue et par le degré de confiance mutuelle entre les systèmes légaux existants ». L'élimination de l'obstacle juridique en ce qui concerne le crime politique est donc capital. Si l'on suit l'argumentation d'Ana Palacio, la confiance mutuelle doit se révéler totale. La possibilité de réserve est incompréhensible, car faire partie de l'Europe, c'est être équivalents en droit : « ou bien nous sommes un groupe d'Etats reconnaissant mututellement que nos systèmes sont équivalents pour garantir les libertés civiques, ou bien l'Union européenne cesse d'exister ${ }^{37} »$.

Le concept de "pays d'origine sûre » qui permet de rejeter ou de refuser de recevoir une demande d'asile au sein de l'Union européenne remonte aux débuts des années 1990, à un moment où précisément se constituent les listes des pays pour lesquels l'Union estime qu'il n'y a généralement pas de risque sérieux de persécutions de quelque sorte que ce soit qui puisse motiver une demande d'asile ${ }^{38}$. Or, c'est dans l'extension, à l'intérieur même des frontières de l'Union européenne, de cette reconnaissance des pays sans risques que l'on trouve les moyens de l'argument espagnol en faveur du refus de considérer une demande d'asile dans un Etat membre de la part d'un ressortissant d'un autre Etat membre. Un Etat membre de l'Union européenne n'est-il pas, par définition, un pays d'origine sûre? En 1997, les Etats membres de l'Union européenne adoptent un protocole soutenu par l'Espagne et amendé au Traité d'Amsterdam. Ce protocole, intitulé Protocole sur le droit d'asile des ressortissants des Etats membres de l'Union européenn $e^{39}$ ", affirme que, à quelques exceptions mineures, les Etats membres n'accepteront aucune demande d'asile de la part de ressortissants d'autres Etats membres. "Vu le niveau de protection des droits fondamentaux et des libertés fondamentales dans les Etats membres de l'Union européenne, ceux-ci sont considérés comme constituant des pays sûrs les uns vis-à-vis des autres pour toutes les questions juridiques et pratiques liées aux affaires d'asile ${ }^{40}$ ». Le " Protocole Aznar », du nom de son rédacteur, José María Aznar, affirme de manière ferme et péremptoire que l'ensemble des Etats-membres de l'Union européenne sont des pays de toute sécurité, dans lesquels il ne peut exister de violations des droits de l'Homme, sans que cela n'entre en contradiction avec les fondements de la construction européenne érigés en principes $^{41}$. C'est la raison pour laquelle toute demande d'asile de la part de citoyens européens à l'intérieur même de l'espace européen est manifestement sans fondement et sera automatiquement rejetée en vertu de ce principe. 
L'argument majeur des autorités politiques espagnoles tient à l'existence d'une contradiction entre la mise en œuvre des dispositions relatives au droit d'asile et à l'extradition en prétextant que des Etats membres de l'Union européenne, accordant l'asile à des terroristes de l'ETA - discours de l'intolérable sanctuaire français, et opposition frontale vis-à-vis des autorités belges dans l'affaire Moreno Garcia -, reconnaissent de la sorte à ces activités délictueuses un caractère politique et introduisent une forme de "prime " à l'impunité pour les terroristes. Les autorités belges qui refusent l'extradition de Moreno-Garcia se retrouvent ainsi, pour les autorités espagnoles, dans une situation intenable mais semblable à celle des autorités françaises du début des années 1980 qui avaient reconnu le droit d'asile pour des activistes italiens - au grand dam des autorités italiennes - et qui, par ailleurs, avaient longuement ajourné le "problème basque » des extraditions de réfugiés et/ou de membres de l'ETA. Une configuration qui illustre cette compromission difficile entre l'extradition qui est admise en dépolitisant l'acte qualifié de "terroriste » et l'asile politique qui, au contraire, tient compte de ce caractère. Dès lors que l'infraction politique est considérée comme un détournement de la finalité de l'asile ouvrant la voie à l'impunité pour les "terroristes " et non plus comme une réserve juridiquement établie visant à protéger des individus contre des extraditions abusives, la question de l'asile politique en Europe a fait un pas vers sa mise à nu ${ }^{42}$. Ce déplacement de la question de l'infraction politique, de sa qualification par le droit vers sa stigmatisation politique, se révèle efficace au point où toute discussion de principe sur la nature d'un délit se joue désormais sans cette question au nom de la "reconnaissance mutuelle " entre Etats membres de l'Union européenne ${ }^{43}$.

En ce sens, le silence particulièrement embarrassé de certains socialistes espagnols à l'époque des "grands procès » des GAL est devenu une solution politique autorisée par défaut. Des deux côtés de la chaîne des Pyrénées et au-delà. La dépolitisation de la violence, en tant que processus global dont le propre est de supporter les irrégularités et les exceptions, est aujourd'hui, plus que jamais, un mouvement européen déterminant. Le brevet d'autosatisfaction que se décernent les Etats membres de l'Union européenne en matière de respect des droits de l'Homme pour justifier la suppression de l'exception à l'extradition pour infraction politique et du droit d'asile pour les ressortissants des Etats-membres est à la fois la preuve et la conséquence de ce mouvement de dépolitisation global qui confond allègrement Etat de droit, démocratie et raison d'Etat pour peu qu'il s'agisse de s'autoriser les moyens de la lutte contre le terrorisme ${ }^{44}$.

29 Le Conseil européen de Cardiff des 15 et 16 juin 1998, sur proposition du Royaume-Uni, a demandé l'évaluation du bénéfice de l'extension de la notion de reconnaissance mutuelle aux décisions en matière pénale. Le 3 décembre 1998, le Plan d'action du Conseil et de la Commission concernant les modalités optimales de mise en œuvre des dispositions du Traité d'Amsterdam relatives à l'établissement d'un espace de Liberté, de Sécurité et de Justice, proposait la mise en route, dans les deux années à venir de l'entrée en vigueur du Traité, d'un processus visant à faciliter le principe de reconnaissance mutuelle des décisions judiciaires. Mais c'est lors du Conseil européen de Tampere, des 15 et 16 octobre 1999, qu'il a été décidé de s'appuyer sur ce principe de reconnaissance mutuelle et d'en faire la pierre angulaire de la coopération judiciaire, tant sur le plan civil que pénal. Trois années plus tard, le 15 janvier 2001, le Programme commun de la Commission et du Conseil établit un ensemble de vingt-quatre mesures 
destinées à mettre en œuvre ce principe de reconnaissance mutuelle des décisions pénales.

Sur la base de ce principe d'un «processus mutuel de reconnaissance» le Mandat d'arrêt européen proposé par la Commission est conçu pour remplacer le système actuel d'extradition en exigeant de chaque autorité juridique nationale (l'autorité juridique d'exécution) de reconnaître, ipso facto, et avec un minimum de formalités, les demandes d'extradition faites par l'autorité juridique d'un autre Etat membre. Ce MAE, adopté lors du Conseil européen de Luxembourg par la décision cadre en date du 13 juin 2002, est-il un outil juridique novateur? Souvent présenté de la sorte, le MAE est bien au contraire le résultat d'un long processus européen, à la fois juridique et politique, de reconstruction du droit extraditionnel en Europe. Cette présentation en terme d'innovation est certes largement tributaire du contexte international faisant suite aux attentats du 11 septembre 2001 sur le sol américain et aux réactions politiques consécutives en Europe. Mais cette présentation tend à masquer combien le MAE n'a pu être pensé et proposé qu'au terme de ce processus de dépolitisation que nous avons souligné précédemment. Autrement dit, le MAE n'a été rendu possible que dans la mesure où l'infraction politique en Europe a été défaite. Le MAE est l'aboutissement de la constitution de cet espace judiciaire européen (proposé par la France et rentabilisé par l'Espagne), de cet espace dans lequel les rétorsions éventuelles de la part d'Etats membres vis-à-vis de la coopération répressive européenne sont rendues impossibles, ou tout du moins particulièrement délicates.

31 Les attentats du 11 septembre 2001 ont créé une fenêtre d'opportunité pour surmonter toute contrainte institutionnelle. Même si la lutte contre le "terrorisme» et, par extension, contre " la criminalité organisée » est le fait d'un grand nombre de policiers, d'agents du renseignement et d'acteurs juridiques, ils sont tous impliqués dans la même (re)définition globale de l'identité européenne. En fait, c'est parce qu'il existe ces réseaux complexes faits de différentes cultures et de pratiques dans la lutte contre le terrorisme que la possibilité de rencontrer un discours unique et commun offre une réelle occasion politique pour tous les acteurs institutionnels impliqués. Comme Zygmunt Bauman l'a précisé, la logique de délimitation de l'identité reste emprisonnée dans la métaphysique de l'absence d'ambiguïté : l'identité est l'un ou l'autre mais avec un milieu exclu ${ }^{45}$. C'est de cette théorisation fermée de l'identité que l'on trouve la possibilité de dire que l'étranger est l'autre et l'autre, l'ennemi ${ }^{46}$. Peu importe que l'on puisse ou non clairement définir qui est un terroriste et qui ne l'est pas. Certains termes servent admirablement bien des fins politiques et normatives malgré le fait qu'ils entravent autant la description que l'explication du phénomène qu'ils pointent. Le terme de « terrorisme » est de ceux-là. Il est formidablement utile pour permettre de réévaluer des valeurs contre d'autres et absorber toute autre analyse ${ }^{47}$. De là, il est possible d'atteindre une circularité parfaite de justification et d'oublier que, à l'origine, la coopération européenne n'était qu'une réunion de Raisons d'Etat contradictoires. En tant que discours fondamental, la «solidarité démocratique» constitue l'identité européenne au nom de laquelle elle opère.

La coopération antiterroriste des démocraties comme acte naturel de solidarité démocratique est une croyance forte, renforcée par les événements des attentats du 11 septembre 2001 aux Etats-Unis et par la mobilisation émotionnelle qui s'est ensuivie. L'idée qu'il existerait un destin commun à toutes les démocraties n'est-elle pas une manière de réduire toute incertitude, y compris à propos de la légitimité de certains 
actes qui, par ailleurs, demeureraient illégitimes? Quand le Président Bush a déclaré la "guerre contre le terrorisme » après les attentats de New York et de Washington, le Président russe Vladimir Poutine a promptement déclaré sa solidarité avec les EtatsUnis et n'a pas manqué de joindre le conflit tchétchène au puzzle de la «lutte globale contre la terreur ». La guerre sale en Tchétchénie est oubliée ${ }^{48}$. Les actions des 45000 soldats russes impliqués en Tchétchénie sont laissées à la seule discrétion du commandant militaire compétent ${ }^{49}$. Et quand le Russian Human Rights Centre Memorial au même titre qu'Amnesty International et le Conseil danois pour les réfugiés (DCR) peuvent prouver que certains soldats sont impliqués dans l'organisation d'escadrons de la mort, de pratiques de tortures et de massacres extrajudiciaires en Tchétchénie, la réponse officielle est tout simplement une fin de non-recevoir. Les preuves, même les plus probantes, ne peuvent être vraies et sont lues comme relevant d'une logique de diffamation intolérable. "Vous devez avoir fait une erreur de continent parce que cette guerre fait partie de la guerre globale des démocraties contre le terrorisme $e^{50} »$. Tout un chacun peut voir combien cette croyance unanimement partagée dans cette narration guerrière d'une solidarité naturelle des démocraties contre le terrorisme tend à rejeter dans l'ombre tout débat politique sur les logiques mêmes de ce discours.

«Il n'y a aucune possibilité pour une réponse soigneusement modulée à moins que nous prenions une certaine distance critique vis-à-vis de cette rhétorique régnante en ce moment. George Bush et Tony Blair ont fait de la guerre contre le terrorisme une puissante arme politique et c'est précisément là que se trouve la racine du problème ${ }^{51}$.

Le discours de la solidarité démocratique n'est certes pas un nouveau discours. C'est l'autorité de ce discours qui relève de la nouveauté. Quel type de répertoire discursif les attentats du 11 septembre ont-ils ouvert? Rien de moins que la possibilité de pouvoir affirmer que la lutte contre le terrorisme ne relève que de la seule capacité des démocraties et que par conséquent la coopération en la matière constitue désormais non seulement un brevet de bonne conduite mais aussi un certificat de bonnes moeurs démocratiques. La coopération produit de la reconnaissance mutuelle, mais elle engendre aussi un phénomène d'occultation majeur. La lutte contre le terrorisme, telle qu'elle se développe depuis les attentats du 11 septembre, introduit un renversement total des perspectives qui fait apparaître les droits fondamentaux comme obstacles à la " sécurité » et leur restriction comme un moyen nécessaire pour protéger les citoyens du terrorisme, défini comme la menace principale contre la « démocratie ${ }^{52}$ ». Mais de quelle démocratie s'agit-il ? L'universalisation de la "démocratie" tend à occulter complètement le problème du contenu et à rendre parfaitement incongru le fait de se poser la question et de faire remarquer que l'on sait de moins en moins ce dont on parle. La question d'un déficit démocratique a été remplacée par la solution d'une solidarité démocratique contre le terrorisme. Soit l'on parle de déficit, soit l'on parle de solidarité. Mais de démocratie pas le moindre mot. 


\section{NOTES}

1.. Cet article est le fruit d'une recherche élaborée dans le cadre du programme de recherches européen ELISE - European Liberty and Security (FP-5). Je remercie Fabienne Brion et Martin Moucheron de m'avoir permis de présenter une ébauche de cet article lors d'un séminaire organisé par leurs soins à l'Ecole de Criminologie de Louvain-La-Neuve (octobre 2005). Je tiens aussi à remercier Philippe Bonditti pour ses nombreux commentaires et je n'oublie pas Pierre Viale pour sa suggestion de titre tout à fait opportune.

2.. Le virus (le terrorisme) s'attaquant à un corps sain (la démocratie) et qui induit le remède (l'antiterrorisme) est une métaphore médicale largement employée dont il faudrait pouvoir rendre compte de manière plus précise. Ce n'est pas l'enjeu de cet article.

3.. Guittet E.-P., « Coopération sans frontières, frontière de la coopération antiterroriste en Europe ", Chantiers politiques, n² , décembre 2004, pp. 43-47.

4.. Reinares F., Jaime-Jimenez O., « Countering Terrorism in a New Democracy : the Case of Spain ", Reinares F. (dir.), European Democracies Against Terrorism. Governmental Policies and Intergovernmental Cooperation, Londres, Ashgate/The Oñati International Institute for the Sociology of Law, 2000, pp. 119-145.

5.. La liste européenne des terroristes a été adoptée en décembre 2001 par l'Union européenne et remise à jour en décembre 2003.

6.. La revendication espagnole portant sur la nécessité de mesures permettant de faciliter l'extradition et de la rendre la plus automatique possible en matière de « terrorisme » s'exprime, dès la fin des années 1970, d'abord dans un cadre bilatéral (la France), avant de devenir un enjeu multilatéral dans le cadre de la constitution d'un espace commun de sécurité (du Traité de Maastricht à Tampere). Voir Alonso Zaldívar C., Castells M., España, fin de siglo, Madrid, Alianza, 1992, p. 210 et suivantes ; Barbé E., La política europea de España, Barcelone, Ariel, 1999 ; Closa C., Heywood P.M. (dir.), Spain and the European Union, Londres, Palgrave, 2004, p. 229 et suivantes.

7.. Sur un marché éditorial saturé de publications diverses et variées en provenance des milieux journalistiques, des experts es terrorisme (policiers, politiques, juges et universitaires compris), peu nombreux sont les travaux où les auteurs dressent la généalogie de ce discours de l'antiterrorisme et instruisent la critique de ces pratiques qui s'inscrivent dans une perception linéaire du devenir et se justifient comme solutions solidaires de cette certitude que les démocraties sont les seules victimes patentées du « terrorisme ». Voir Bonditti P., Guittet E.-P., « Etat des analyses sur les politiques antiterroristes depuis le 11 septembre 2001 : modalités et fonctions de l'exceptionnalisme », Paris, Cultures \& Conflits/Délégation aux Affaires Stratégiques (DAS), Ministère de la Défense, décembre 2002, disponible à l'adresse suivante :

http://www.defense.gouv.fr/sites/das/dossiers/ politiques_antiterroristes_depuis_le_11_septembre_2001

8.. Nous développons ce point dans notre thèse : La Genèse de la coopération antiterroriste en Europe et l'implication de l'Espagne dans la (re)définition de l'identité européenne. De la raison d'Etat à la raison de la gouvernementalité européenne?

9.. Au Pays basque.

10.. Province basque de la Viscaya 
11.. Province basque du Guipuzcoa.

12.. Guittet E.-P., Raison et déraison d'Etat : les GAL (Groupes Antiterroristes de Libération) 1983-1987, Mémoire pour le DEA de Sociologie Politique, Université Paris-X Nanterre, septembre 2000 ; Woodworth P., Dirty War Clean Hands. ETA, the GAL and Spanish Democracy, New Haven/Londres, Yale University Press, 2002.

13. Déclaration du Général José Antonio Saenz de Santamaría publiée dans l'hebdomadaire espagnol La Razon dans son édition du 19 novembre 2001. Voir aussi ses déclarations dans le quotidien madrilène El País dans son édition du 24 février 1995 : "Dans la lutte antiterroriste, il y a des choses que l'on ne doit pas faire. Si on les fait, il ne faut pas le dire. Si ça se dit, il faut tout nier ».

14.. Le ministre de l'Intérieur français Raymond Marcellin signe le décret de «nullité de l'association étrangère dite "Euzkadi Ta Azkatasuna" » le 8 octobre 1972. 15.. Ménage G., « Face aux terrorismes. 1981 - 1986. Action directe, Corse Pays basque ", L'ÆFil du pouvoir, t. 2, Paris, Fayard, 2000, p. 15 et suivantes ; Cassan P., Le Pouvoir français et la question basque (1981-1993), Paris, l'Harmattan, 1997, p. 201 et suivantes.

16. Pour mémoire, voir la déclaration du Premier ministre Pierre Mauroy au « Club de la Presse » d'Europe 1, le 8 juin 1981 : « La France est une terre d'asile pour tous ceux qui sont des condamnés politiques et doit rester fidèle à son histoire et à son image "; voir aussi la position du ministre de l'Intérieur Gaston Deferre publiée dans le Nouvel Observateur dans son édition du 18 juillet 1981 : « peut-être parce que j'ai vécu la clandestinité, je ressens très fortement qu'extrader est contraire à toutes les traditions de la France, surtout lorsqu'il s'agit comme là d'un combat politique ».

17. Voir le seul communiqué écrit des GAL retrouvé dans les poches de Segundo Marey enlevé par les GAL et libéré dix jours plus tard le 14 décembre 1983 parce que ses ravisseurs l'avaient confondu avec un membre de l'ETA : «1.- Chaque assassinat des terroristes aura la réponse nécessaire ; pas une seule des victimes permanettra sans réponse. 2.Nous manifestons notre idée d'attaquer les intérêts français en Europe puisque son Gouvernement est responsable d'accueillir et permettre actuer les terroristes dans territoire impunément. Aucune personnalité et aucun objectif de l'économie Française va être sûr à présent ». Nous reproduisons ici cet extrait du communiqué tel qu'il est porté dans le dossier d'instruction de l'enlèvement de Segundo Marey, y compris dans son français approximatif.

18.. Reinares F., « Fundamentos para una política gubernamental antiterrorista en el contexto de regímenes democráticos », Sistema, n¹32-133, 1996, p. 126 et suivantes.

19.. Ménage G., op. cit., p. 402 et p. 506.

20.. « Galos ", terme péjoratif pour parler des Français.

21.. Tribunal Constitucional, Recurso de Amparo $n^{\circ} 3721 / 98$, BOE, num. 83 Suplemento, 6 avril 2001, p. 8 et suivantes. La défense est de prétendre que la création des GAL relevait d'un " état de nécessité ».

22. Les articles du Canard Enchaîné du 26 mars et 27 juin 1984 montrent que des membres des GAL ont été retrouvés en possession de nombreuses photographies, remises par les réfugiés à la sous-préfecture de Bayonne. Dans son livre, JeanMarc Dufourg, inspecteur aux RG, atteste que les dossiers concernant les réfugiés basques étaient remis par les Renseignements Généraux à la police espagnole qui les transmettait aux GAL. Dufourg J.-M., Section Manipulation. De l'antiterrorisme à l'affaire Doucé, Paris, Michel Lafon, 1991. Les suspicions à l'égard de fonctionnaires français impliqués à un degré ou un autre dans les GAL sont nombreuses, mais ce n'est qu'avec 
les déclarations en 1994 du policier espagnol José Amedo Foucé que les présomptions se précisent et que des noms apparaissent à la fois dans la presse (française et espagnole) mais aussi devant les tribunaux espagnols, à la faveur des témoignages de plus en plus abondants. Dans ses déclarations de 1994, Amedo Foucé accable les services français de police en affirmant avoir eu des contacts à de nombreuses reprises avec des fonctionnaires français, cela dès 1982, et avoir reçu l'ordre de ses supérieurs de prendre contact avec ceux-ci et de payer pour les renseignements obtenus.

23.. Bigo D., Guittet E.-P., « Vers une nord-irlandisation du monde ? ", Cultures \& Conflits, $\mathrm{n}^{\circ} 56,2004, \mathrm{p} .173$ et suivantes.

24.. Intervention de Viviane Reding, rapporteur du Parlement européen sur la lutte contre le terrorisme au sein de l'UE, lors de la conférence parlementaire intitulée " Les démocraties face au terrorisme ", Strasbourg, 14-16 octobre 1998. Propos reproduits dans : Parlement européen, Commission des questions juridiques et des droits de l'Homme, Compte-rendu de la conférence parlementaire sur les démocraties européennes face au terrorisme, AS/Jur (1999) 11, 22 février 1999, p. 68.

25.. La Conférence intergouvernementale a officiellement débuté le 29 mars 1996 à Turin. Un premier rapport fut débattu à Florence les 21 et 22 juin 1996.

26.. Cortes Generales, Diario de Sesiones del Congreso de los Diputados, Comisión Justicia e Interior Sesión nº 12, 23 février 1994, p. 3801.

27.. Barrionuevo J., 2001 días en Interior, Barcelona, Ediciones B, 1997, p. 173.

Barrionuevo rappelle la demande d'extradition à l'encontre de José Arteche Gonzalez et Salvador Ormaza Corral qui date de janvier 1983. Ils sont extradés vers l'Espagne en juillet 1984. Ils sont les premiers etarras de la branche politico-militaire de l'ETA (VIII ${ }^{\mathrm{e}}$ assemblée) à être extradés vers l'Espagne. C'est aussi la première fois depuis 1945 que le gouvernement belge accorde une extradition de membres d'une organisation clandestine. « Las autoridades de Bruselas conceden la primera extradición por terrorismo », El País, 15 juillet 1984.

28.. Parlement européen. Résolution sur l'extradition de deux militants présumés de l'ETA, Journal Officiel C 065, 4 mars 1996, p. 0160.

29.. Closa C., Heywood P.M., op. cit., pp. 6-30.

30.. Propos de José María Aznar, Conférence de presse après une rencontre avec le Président français Jacques Chirac, Paris, juin 1996.

31.. Westendorp C., The Wall Street Journal dans son édition du 20 février 1996. Article disponible auprès de Oficina de Informacion Diplomatica (OID), Ministerio de Asuntos Exteriores, Textos y Documentos de la Politica Exterior Espanola, Madrid, 1996.

32.. Bribosia E., Weyembergh A., «Extradition et asile : vers un espace judiciaire européen? ", Revue belge de droit international, n¹, 1997, p. 76 et suivantes.

33.. Convention relative à l'extradition entre les Etats membres de l'Union européenne, JOCE, $n^{\circ} \mathrm{C} 313$ en date du 23 novembre 1996 ; rapport explicatif, JOCE, $n^{\circ} \mathrm{C} 191$ en date du 23 juillet 1997.

34.. Suivant l'analyse de Weyembergh A., «La coopération pénale européenne face au terrorisme : rupture ou continuité ? ", Bannelier K., Corten O., Christakis T. et Delcourt B. (dir.), Le Droit international face au terrorisme après le 11 septembre 2001, Paris, Pédone/ CEDIN - Paris I, Cahiers internationaux, n ${ }^{\circ} 17,2002$, p. 287.

35.. Article 1 de la Convention de 1996.

36.. Bribosia E., Weyembergh A., op. cit., p. 87.

37.. Oficina de Informacion Diplomatica (OID), Ministerio de Asuntos Exteriores, Textos y Documentos de la Politica Exterior Espanola, Madrid, 1996. 
38.. Guild E, Niessens J., The Developping Immigration and Asylum Policies of the European Union, The Hague, Kluwer Law International, 1996, pp. 177-180.

39.. Protocole sur le droit d'asile pour les ressortissants des Etats membres de l'Union européenne, Traité d'Amsterdam, 1997, Protocole annexé au Traité constituant la Communauté européenne.

40.. Ibid.

41.. « Attachement aux principes de la liberté, de la démocratie et du respect des droits de l'Homme et des libertés fondamentales et de l'Etat de droit », préambule du Traité sur l'Union européenne, Maastricht, 7 février 1992.

42.. Entretien avec R. Errera, septembre 2005.

43.. Voir l'édition spéciale du numéro de Cultures\&Conflits, « De Tampere à Séville : Rapport sur la sécurité européenne », n46, été 2002. Voir également Apap J., Incerti M., Trust and Co-operation in Judicial, Extradition, Immigration and Asylum Matters, Bruxelles, CEPS, mars 2002.

44.. Guild E., « Looking Towards the Future », in Brouwer E., Catz P., Guild E., (dir.), Immigration, Asylum and Terrorism. A Changing Dynamic in European Law, Nijmegen, Recht \& Samenleving, Instituut voor Rechtssociologie / Centrum voor Migratierecht, vol.19, 2003, p.176 et suivantes.

45.. Voir Bauman Z., Modernity and Ambivalence, Cambridge, Polity Press, 1992. Voir aussi Beck U., Democracies without Enemies, Cambridge, Polity Press, 1998, p. 138.

46.. Voir Cultures \& Conflits, "Suspicion et exception », n58, 2005 et plus particulièrement l'article de Walker R.B.J., « L'international, l'impérial, l'exceptionnel », pp. 13-51.

47.. Bigo D., « Does the War Put an End to Political Violence of Clandestine Organisations? A European Point of View », contribution à la recherche de l'équipe française ELISE, mars 2004.

48.. « Poutine et le terrorisme », Le Monde, le 27 décembre 2001 ; voir également la réunion entre Bush, Poutine et Jiang Zemin en octobre 2001.

49.. Dimitri V. Trenin souligne que « tant que la guerre continuera en Tchétchénie, le Haut Commandement militaire aura un argument de poids et un ascendant politique fort contre toute réforme militaire radicale " (" as long as the war in Chechnya continues, the High Military Command will have a powerful argument and political clout against a radical military reform "), Carnegie Endowment for International Peace, Policy Brief, $n^{\circ} 28$, novembre 2003.

50.. Voir l'entrevue de Vladimir Rouchailo, secrétaire du Conseil de sécurité russe, dans le quotidien français Libération en date du 26 décembre 2001.

51.. Ackerman B., " Don't Panic », London Review of Books, vol.24, n³, 7 février 2002.

52.. Voir la contribution de Tsoukala A. pp. 35-50 dans ce numéro de Cultures \& Conflits.

\section{RÉSUMÉS}

La solidarité naturelle des sociétés démocratiques européennes contre le terrorisme est un discours qui ne peine pas à s'imposer comme vrai tant il s'énonce sur le mode de l'évidence. Evidence de la menace qui produit l'évidence de la réponse : une coopération antiterroriste de 
tous contre un ennemi communément admis. En analysant ce discours d'autorité, il s'agit de retrouver les conditions de félicité de cette adéquation performative entre "démocratie » et « coopération » antiterroriste. En revenant sur l'histoire, les discours et les pratiques officiels et clandestins de l'antiterrorisme espagnol et leur imposition au niveau européen, l'auteur montre combien la «solidarité démocratique » constitue l'identité européenne au nom de laquelle elle opère.

The natural solidarity against terrorism between European democratic societies, taken as a discourse, has no difficulties to assert itself given its "obviousness". The obviousness of the threat indeed makes the obviousness of the reply: everyone cooperates against the one enemy everyone admits being such. If we are to analyze this discourse of authority, we need the propitious conditions of this performative adequacy between "democracy " and antiterrorist " cooperation ». By recalling official and clandestine History, discourses and practices, the author shows how democratic solidarity composes the European identity in the name of which this solidarity takes effect.

\section{INDEX}

Mots-clés : anti-terrorisme, démocratie, identité

Index géographique : Espagne

Thèmes : ETA

\section{AUTEUR}

\section{EMMANUEL-PIERRE GUITTET}

Emmanuel-Pierre Guittet est doctorant en sociologie politique à l'Université Paris-X Nanterre, chargé de recherche au Centre d'Etudes sur les Conflits, enseignant à l'IEP de Paris et Junior Researcher dans les programmes ELISE et CHALLENGE ( $5^{\mathrm{e}}$ et $6^{\mathrm{e}}$ PCRD de la Commission européenne). 